\title{
Preparation and characterization of regenerated Bombyx mori silk fibroin fiber with high strength
}

\author{
Z. H. Zhu, K. Ohgo, T. Asakura* \\ Department of Biotechnology, Tokyo University of Agriculture and Technology, Koganei, Tokyo 184-8588, Japan
}

Received 1 October 2008; accepted in revised form 10 November 2008

\begin{abstract}
Regenerated Bombyx mori silk fibers were spun from hexafluoro-iso- propanol solution of silk fibroin sponge in methanol used as a coagulant solvent and then elongated in water. The stress-strain curves of the regenerated fibers changed dramatically depending on the draw ratio and the structure was studied by ${ }^{13} \mathrm{C} \mathrm{CP/MAS} \mathrm{NMR} \mathrm{and} \mathrm{X-ray} \mathrm{diffraction} \mathrm{meth-}$ ods. The patterns of ${ }^{13} \mathrm{C} \mathrm{CP} / \mathrm{MAS}$ NMR spectra of two regenerated fibers with different draw ratios $(1 \times$ and $3 \times)$ and native silk fiber are all $\beta$-sheet structure although the fraction of random coil/distorted $\beta$-turn decreases in the order of $1 \times, 3 \times$ and native fiber gradually. On the other hand, azimuthal scans of their X-ray fiber patterns changed remarkably with increasing the draw ratio. This indicates that long-range orientation of the fibroin chain changes remarkably during the drawing process, but the short-range local structure does not change significantly. Regenerated silk fiber with a draw ratio of $3 \times$ is a fiber with high strength which is comparable with that of natural silk fiber. The regenerated fiber is also more degradable than natural silk fiber in enzyme solution in vitro.
\end{abstract}

Keywords: biopolymers, regenerated silk fiber, hexafluoro-iso-propanol

\section{Introduction}

Silk proteins are of practical interest because of their excellent intrinsic properties utilizable in biotechnological and biomedical fields [1]. It is advantageous to focus on the regeneration of natural silks and their structural information in the spinning process because these can be used as a benchmark for evaluating the success of the spinning process. Hexafluoro-iso-propanol (HFIP) [2-7], hexafluoroacetone hydrate [8] and formic acid [9] have been used as useful solvents for the regeneration of the silk fibroins. Since the mechanical properties of the regenerated silk fiber depend on the condition of the preparation strongly, the nature of the solvent and the post-spinning treatments applied to the fibers may be important factors, affecting the overall quality and properties of the fibers produced.
One of the requirements for scaffolds for tissue engineering is degradability to be metabolized by the host once its repair function has been completed. Silk fiber is well-known as a hardly degradable material in vivo, but the regenerated silk fiber might be a candidate of biodegradable fiber. The regenerated silk fiber with high strength and high degradability will undoubtedly lead the development of new biocompatible scaffolds made of silk. In this paper, we attempt to show that HFIP may be a solvent of choice for the preparation of regenerated silk fibroin fibers. A process for the artificial spinning of Bombyx mori silk fibroin solution in HFIP will be confirmed. Solid-state ${ }^{13} \mathrm{C}$ nuclear magnetic resonance (NMR) and wide-angle X-ray diffraction (WAXD) will be used for characterization of the regenerated silk fibers obtained here. The biodegradation of the regenerated fibers will

*Corresponding author, e-mail: asakura@cc.tuat.ac.jp

(C) BME-PT and GTE 
be examined by immersing the fibers in enzyme solution.

\section{Materials and methods}

\subsection{Preparation of regenerated Bombyx mori silk fibroin fiber}

Bombyx mori cocoons were degummed according to the method reported previously [8] and then the degummed silk fiber was dissolved in $9 \mathrm{M} \mathrm{LiBr}$ solution to a concentration of $10 \% \mathrm{w} / \mathrm{v}$ at $40^{\circ} \mathrm{C}$ for 1 hour and then dialyzed against deionized water for 4 days at $4^{\circ} \mathrm{C}$. HFIP was of analytical grade (Wako Pure Chemical Industries Ltd., Japan) and used for the solvent of the silk fibroin. The lyophilized silk sponge was dissolved in HFIP for 2 days, yielding a $12 \mathrm{w} / \mathrm{v} \%$ solution. The HFIP solution was extruded through a stainless steel spinneret with $0.45 \mathrm{~mm}$ inner diameter using syringe pump into the methanol coagulation bath at room temperature. The extruded filament was soaked in the methanol bath over $3 \mathrm{~h}$ to allow the HFIP to diffuse from the fiber before drawing. The drawing process of as-spun silk fiber was performed in distilled water bath using a manual drawing method at $40^{\circ} \mathrm{C}$. Draw ratios of 2.0 and 3.0 to its original length were used to examine the drawing effect on the structure and properties of the regenerated fibers. The fibers after the post-spinning treatments were immobilized on the bobbins to prevent recoil and dried overnight at room temperature. The surface morphology of samples was observed with VE-7800 scanning electron microscope (KEYENCE, Japan).

\subsection{Characterization of regenerated silk fibers}

The ${ }^{13} \mathrm{C}$ solid-state CP/MAS NMR spectra of the silk fibers were obtained on a Chemagnetics CMX $400 \mathrm{MHz}$ spectrometer using a cross-polarization pulse sequence and with magic-angle spinning at $9 \mathrm{kHz}$. The experimental conditions were as follows: contact time of $1 \mathrm{~ms}$, pulse delay of $3 \mathrm{~s}$. The $\mathrm{X}$-ray diffraction analyses of the fibers were performed using WAXD experiments on the ultra $X 18$ Rigaku rotating anode generator with graphite monochromatized $\mathrm{CuK}_{\alpha}$ radiation and cylindrical IP (R-AXIS Rapid). The voltage and current of the $\mathrm{X}$-ray source were $50 \mathrm{kV}$ and $250 \mathrm{~mA}$, respec- tively. Well-aligned fiber bundles of Bombyx mori native fibroin fibers or the regenerated fibroin fibers from HFIP system were mounted vertically at the exit of the collimator. The mechanical properties of the native and the regenerated silk fibers were measured using an EZ-Graph tensile testing machine (SHIMADZU Co. Ltd.) at room temperature with $5 \mathrm{~N}$ load cell. The rate of crosshead was $10 \mathrm{~mm} / \mathrm{min}$ on samples of $25 \mathrm{~mm}$ length. Each value was the average of 10 measurements.

\subsection{In vitro enzymatic degradation}

The degradation of the regenerated silk fibroin fibers was evaluated using protease XIV (EC 3.4.24.31, Sigma-Aldrich) with an activity of $4 \mathrm{U} / \mathrm{mg}$. The fibers were immersed in phosphate buffer saline (PBS, $\mathrm{pH}$ 7.4) containing protease $(8 \mathrm{U} / \mathrm{ml})$ at $37^{\circ} \mathrm{C}$. After the specific time, the fibers were washed with PBS and distilled water. The enzyme solution was replaced with newly prepared solution every $24 \mathrm{~h}$. For control, the silk fibers were immersed in PBS without enzyme. The degree of degradation was estimated by SEM pictures.

\section{Results and discussion}

\subsection{Morphology and mechanical properties}

The silk sponge obtained from $2 \mathrm{w} / \mathrm{v} \%$ aqueous solution of silk fibroin was completely dissolved in HFIP for 2 days. Since the silk sponge used here takes random coil form and there are many holes within the sponge, it is relatively easy to resolve it in the solvent. The regenerated fibers exhibited a uniform and circular cross-sectional shape as well as a dense morphology. The fiber diameter could be varied from $50 \mu \mathrm{m}(1 \times)$ to $30 \mu \mathrm{m}(3 \times)$ with increasing the draw ratio although the circular shape remains during drawing. The mechanical properties of the regenerated silk fibers were improved by increasing the draw ratio. Figure 1 shows a comparison between the stress-strain curves of the regenerated silk fibers with different draw ratios $(1 \times-3 \times)$ together with the native Bombyx mori silk fiber. The as-spun fiber $(1 \times)$ shows the curve with low tensile strength and high elongation at break. The tensile strength of the fiber increases dramatically with increasing draw ratio. Namely, for asspun fiber, the tensile strength [MPa] was $140 \pm 18$, but the strength increased up to $162 \pm 25$ at a draw 


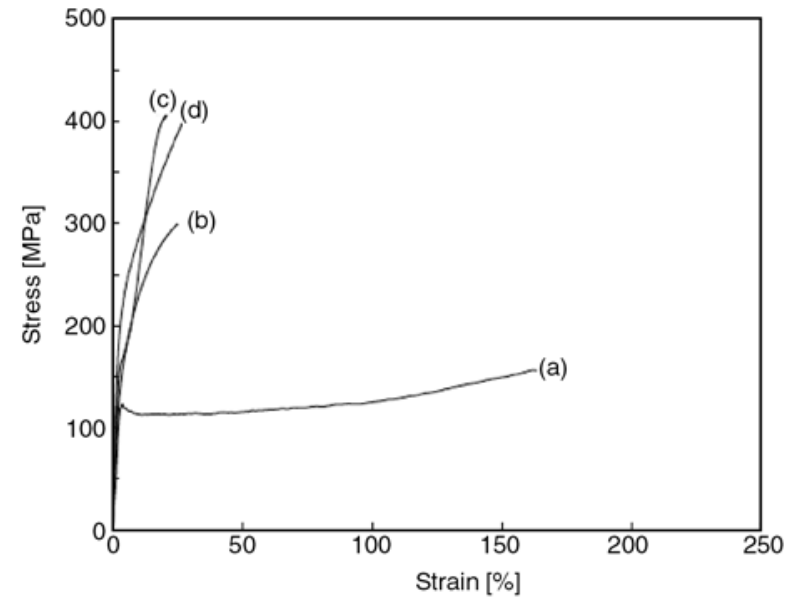

Figure 1. Stress-strain curves of wet-spun regenerated silk fibers with various draw ratios: (a) as-spun, (b) $2 \times$, (c) $3 \times$ and (d) native silk fiber. All curves are the average of 10 measurements.

ratio of $3 x$. The latter value is almost the same or slightly higher compared with the strength of native silk, $398 \pm 51$. The value of tensile strength of native Bombyx mori fiber is slightly lower than the value reported previously. One of the reasons is due to the native fiber with silk sericin used here which is slightly lower than the value of silk fiber without sericin [10]. In addition, difference of the race of silkworm and part of the fiber taken from the cocoon might be the origin of the difference. However, we can compare the values of tensile strength between native and regenerated silk fibers because the experimental condition is the same between them. The elongation at break [\%] changes from $162 \pm 25(1 \times)$ to $21 \pm 3(3 \times)$, where the latter value is similar to the value of the native fiber, $27 \pm 0.8$. The initial modulus [GPa] changes from 5.6 $\pm 1.8(1 \times)$ to $7.3 \pm 0.2(3 \times)$, and the latter is smaller than the corresponding value of the native fiber, 9.8 \pm 0.6 .

\subsection{Solid state NMR analysis}

The conformation-dependent ${ }^{13} \mathrm{C}$ NMR chemical shift and also the line-width have been successfully used to distinguish the secondary structure among Silk I, Silk II, $\alpha$-helix, 3 -helix and random coil [11]. Figure 2 shows ${ }^{13} \mathrm{C} \mathrm{CP/MAS} \mathrm{NMR} \mathrm{spectra} \mathrm{of}$ the regenerated silk fibers prepared from the HFIP solution together with that of native Bombyx mori silk fiber. The peak assignments have been reported previously [4]. The peak positions of the $C \alpha$ and $\mathrm{C} \beta$ carbons from Ala and Ser residues indicate clearly that these samples take $\beta$-sheet structure. In

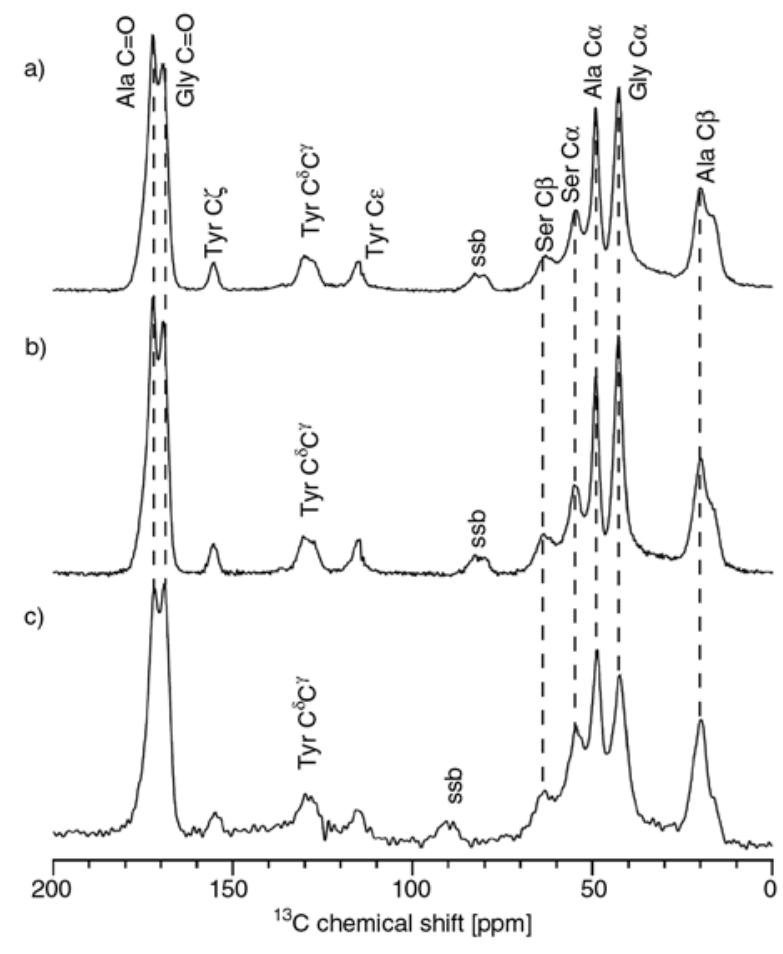

Figure 2. ${ }^{13} \mathrm{C}$ CP/MAS NMR spectra of wet-spun regenerated silk fibers with various draw ratios: (a) asspun, (b) $3 \times$ and (c) native silk. Spinning sidebands are indicated by ssb.

addition, gradual decrease in the fraction of random coil/distorted $\beta$-turn peak observed as shoulder at $16.8 \mathrm{ppm}$ [4], was observed for the Ala $\mathrm{C} \beta$ peak in the order of $1 \times, 3 \times$ and native fiber.

Methanol is known as a coagulant and an inducer of $\beta$-sheet for the silk solutions such as aqueous, formic acid, and HFA solutions. In this study, methanol also induced $\beta$-sheet of silk fibroin chain for the HFIP solution. Simultaneously HFIP is dispersed in the methanol bath during wet-spinning process. Namely, no trace of the HFIP peaks was observed in the ${ }^{13} \mathrm{C}$ CPMAS NMR spectrum of as spun fiber (Figure 2 (a)).

\subsection{WAXD analysis}

WAXD fiber patterns of the regenerated silk fibroin fibers are shown in Figure 3, along with that of native silk fiber. As the draw ratio increased from $1 \times$ to $3 \times$, the fiber shows an increase of the preferential orientation along the fiber axis (Figure $3 a$ and $3 b$ ). The native silk fiber shows a highly orientated diffraction pattern with characteristic arcs having some degree of diffraction intensity (Figure 3c). In order to determine the crystalline characteristics and molecular orientation of the 
(a)

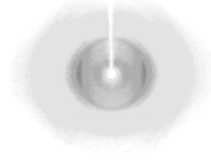

(b)

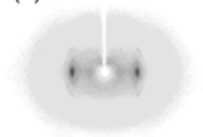

(c)
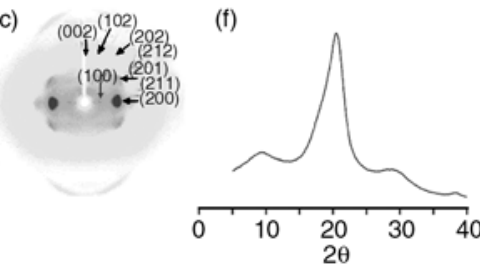

(d)

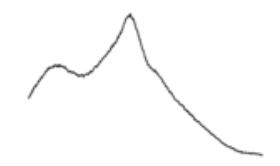

(e)

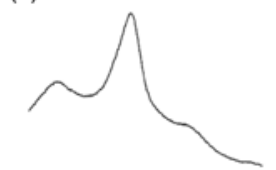

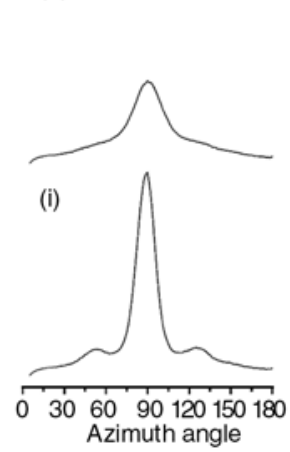

(g)

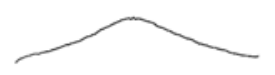

(h)
Figure 3. X-ray fiber patterns (a-c), diffractograms (d-f) and azimuthal scans $(\mathrm{g}-\mathrm{i})$ of the regenerated silk fibers: (a, d, g) as-spun, (b, e, h) $3 \times$ and (c, f, i) native silk fibers.

regenerated fibers more precisely, $2 \theta$ and an azimuthal scan were performed. Figure $3 d-3 f$ shows the X-ray diffractograms of the fibers with different draw ratios. Regardless of the draw ratio, the regenerated fibers showed characteristic $\beta$-sheet crystalline spacings which are identical to those of the native silk. This is consistent with the result from ${ }^{13} \mathrm{C} \mathrm{CP} / \mathrm{MAS}$ NMR spectra that the short-range local structure in the regenerated silk fiber does not change if the drawing ratio changes. To investigate the long-range molecular orientation in crystalline region, an azimuthal scan was performed on XRD fiber patterns (Figure 3g-3i). The crystalline orientation was examined at $2 \theta=$ $19-21^{\circ}$, which well reflects a $\beta$-sheet crystalline. The full width at half-maximum (fwhm) of the peaks taken from the azimuthal intensity scan was $15^{\circ}$ for the native fibers, and 70 and $30^{\circ}$ for the regenerated fibers of $1 \times$ and $3 \times$, respectively. Thus the degree of the long-range orientation is ordered as; native fiber $>$ the regenerated fiber with a draw ratio of $3 x>>$ as-spun fiber[KO1] (1x).

\subsection{Enzymatic degradation}

In vitro degradation of the regenerated fibers incubated in protease was observed. Figure 4 shows change in the mechanical property before and after degradation (5 days after degradation) together with SEM pictures of the regenerated silk fibers
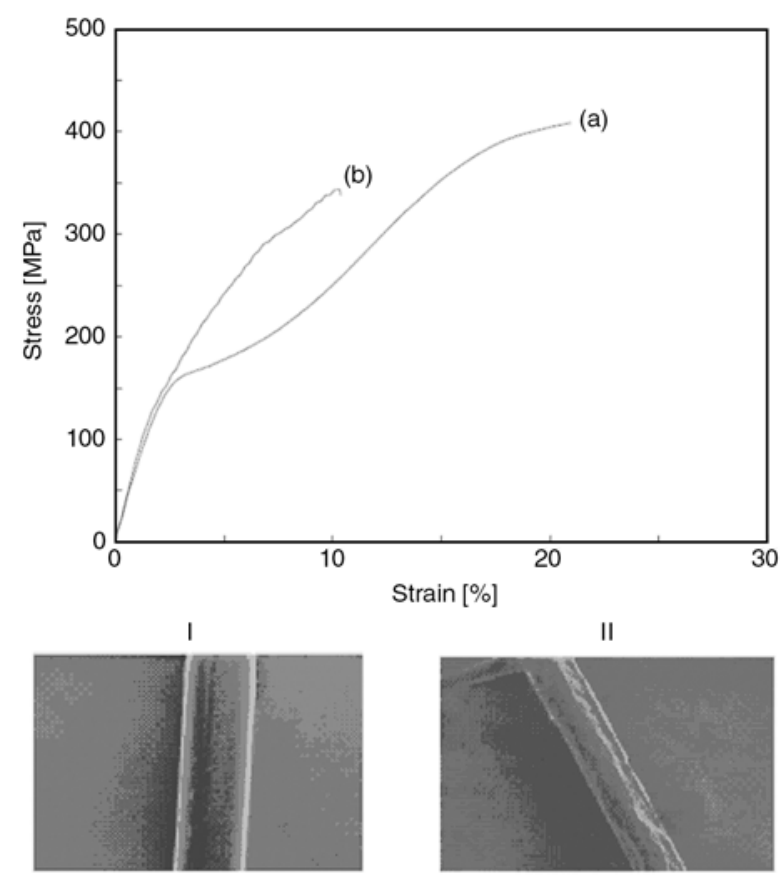

$20 \mu \mathrm{m}$

Figure 4. Stress-strain curves of wet-spun regenerated silk fibers with draw ratio ( $3 \times$ ) before (a) and after degradation (5days) (b). All curves are the average of 10 measurements. The SEM pictures of the fibers before (I) and after degradation (5days) (II) are also shown.

$(3 \times)$. The roughness of the surface was started on 5 days in the case of $3 \times$ drawn fiber as shown in Figure 4 II. The tensile strength [MPa] and elongation at break [\%] decrease by degradation by protease; $333 \pm 51$ and $11 \pm 4$ compared with the corresponding values (a) before degradation, $408 \pm 80$ and $21 \pm 3$, respectively. The initial modulus [GPa] is almost the same, $7.3 \pm 0.2$ and $7.4 \pm 0.4$ for before and after degradation, respectively.

On the other hand, there are no changes in the surface morphology for native fiber in enzyme solution even after 9 days.

The difference of enzymatic degradation between the regenerated silk and the native silk fibers likely reflects differences in the long-range molecular orientation in crystalline region. This may be attributed to the mechanism that low molecular orientation allows the penetration of water molecules and protease into the crystalline domain and then the degradation is accelerated [12]. The degradation rate of scaffolds is an important parameter for sutures and scaffolds for tissue engineering to match new tissue in growth. 


\section{Conclusions}

The regenerated silk fibroin fibers with a comparable strength with natural silk fibers were obtained with HFIP as a spinning solvent and methanol as a coagulant solvent. The combination with the useful mechanical properties and biocompatibility of the regenerated fibers offer new options such as suture, artificial blood vessels with bladed fibers and other scaffolds made of fibers.

\section{Acknowledgements}

The authors thank Dr. Keichi Noghchi at Tokyo University of Agriculture and Technology for assistance with the Xray diffraction measurements. T. A. acknowledges support from Grants-in-Aid for Scientific Research (S) (No. 18105007), Japan.

\section{References}

[1] Asakura T., Kaplan D. L.: Silk production and processing. in 'Encyclopedia of agricultural science' (ed.: Arutzen C. J.) Academic Press, New York, Vol 4, 1-11 (1994).

[2] Trabbic K. A., Yager P.: Comparative structural characterization of naturally- and synthetically-spun fibers of Bombyx mori fibroin. Macromolecules, 31, 462471 (1998).

[3] Liivak O., Blye A., Shah N., Jelinski L. W.: A microfabricated wet-spinning apparatus to spin fibers of silk proteins. Structure-property correlations. Macromolecules, 31, 2947-2951 (1998).

[4] Zhao C. H., Yao J. M., Masuda H., Kishore R., Asakura T.: Structural characterization and artificial fiber formation of Bombyx mori silk fibroin in hexafluoro-iso-propanol solvent system. Biopolymers, 69, 253-259 (2003).
[5] Kim H. J., Kim U-J., Gordana V-N., Min B-H., Kaplan D. L.: Influence of macroporous protein scaffolds on bone tissue engineering from bone marrow stem cells. Biomaterials, 25, 4442-4452 (2005).

[6] Drummy L. F., Phillips D. M., Stone M. O., Farmer B. L., Naik R. R.: Thermally induced alpha-helix to betasheet transition in regenerated silk fibers and films. Biomacromolecules, 6, 3328-3333 (2005).

[7] Ha S. W., Asakura T., Kishore R.: Distinctive influence of two hexafluoro solvents on the structural stabilization of Bombyx mori silk fibroin protein and its derived peptides: C-13 NMR and CD studies. Biomacromolecules, 7, 18-23 (2006).

[8] Yao J. M., Masuda H., Zhao C. H., Asakura T.: Artificial spinning and characterization of silk fiber from Bombyx mori silk fibroin in hexafluoroacetone hydrate. Macromolecules, 35, 6-9 (2002).

[9] Um I. C., Kweon H., Lee K. G., Ihm D. W., Lee J. H., Park Y. H.: Wet spinning of silk polymer - I. Effect of coagulation conditions on the morphological feature of filament. International Journal of Biological Macromolecules, 34, 89-105 (2004).

[10] Altman H. G., Diaz F., Jakuba C., Calabro T., Horan R. L., Chen J.. Lu H., Richmond J., Kaplan D. L.: Silk-based biomaterials. Biomaterials, 24, 401-416 (2003).

[11] Asakura T., Yao J. M., Yamane T., Umemura K., Ulrich A. S.: Heterogeneous structure of silk fibers from Bombyx mori resolved by C-13 solid-state NMR spectroscopy. Journal of the American Chemical Society, 30, 8794-8795 (2002).

[12] Plaza G. R., Corsini P., Perez-Rigueiro J., Marsano E., Guinea G. V.: Effect of water on Bombyx mori regenerated silk fibers and its application in modifying their mechanical properties. Journal of Applied Polymer Science, 109, 1793-1801 (2008). 